\title{
APPROXIMATION OF FUNCTIONS BY CONVEXITY PRESERVING CONTINUOUS LINEAR OPERATORS
}

\author{
JEAN TZIMBALARIO
}

\begin{abstract}
In this note, extension of the ideas used by Korovkin, Bojanic and Roulier, Karlin and Studden, Lorentz and Schumaker are studied in order to treat various aspects of approximation of continuous functions by operators which preserve generalized convexity in the sense of Karlin and Studden, and Ziegler.
\end{abstract}

There is an intensive study of the approximation by positive linear operators. The fundamental ideas for direct estimates appear in the book of P. P. Korovkin [4]. The main ideas for the saturation problem are concentrated in DeVore's book [3]. S. Karlin and W. J. Studden [5] give a complete study of the Chebyšev systems and their connection with the concept of approximation of functions. The problem of saturation of positive operators in this general context was studied lately by G. G. Lorentz and L. L. Schumaker [6].

In this note we wish to announce that one may extend the ideas used by P. P. Korovkin [4], R. Bojanic and J. A. Roulier [2], S. Karlin and W. J. Studden [5], and G. G. Lorentz and L. L. Schumaker [6] to treat the various aspects of approximation of continuous functions by operators which preserve generalized convexity in the sense of Z. Ziegler [7] and S. Karlin and W. J. Studden [5].

Let $w_{0}, \ldots, w_{l+2}(l \geq 0)$ be a finite sequence of continuous strictly positive functions in an open interval $(a, b)$ with $w_{j} \in C^{l+2-j}(a, b), j=$ $0, \ldots, l+2$. Let $D_{0} f(x), \ldots, D_{l+2} f(x)$ be a sequence of operators defined by

$$
D_{j} f(x)=\left[f(x) / w_{j}(x)\right]^{\prime}
$$

also let $D^{j}$ be the operators

$$
D^{j}=D_{j} D_{j-1} \cdots D_{0}, \quad j=0,1, \ldots, l+2,
$$

and

$$
\bar{D}^{j}=D^{j-1} / w_{j}, \quad j=1, \ldots, l+2 .
$$

Define the functions

Received by the editors October 3, 1974.

AMS (MOS) subject classifications (1970). Primary 41 A35.

Copyright $\odot 1975$, American Mathematical Society 


$$
\begin{aligned}
& v_{0}(x)=w_{0}(x) \\
& v_{1}(x)=w_{0}(x) \int_{a}^{x} w_{1}\left(\zeta_{1}\right) d \zeta_{1}, \\
& \quad \cdot \\
& \quad \cdot \\
& v_{l+2}(x)=w_{0}(x) \int_{a}^{x} w_{1}\left(\zeta_{1}\right) d \zeta_{1} \int_{a}^{\zeta_{1}} w_{2}\left(\xi_{2}\right) d \zeta_{2} \cdots \int_{a}^{\zeta} w_{l+2}\left(\zeta_{l+2}\right) d \zeta_{l+2} .
\end{aligned}
$$

It is known that $v_{0}, \ldots, v_{j-1}$ form a fundamental set of solutions of the differential equation $\bar{D}_{j} v=0$ and $\bar{D}_{j} v_{j}=1 . v_{0}, \ldots, v_{l+2}$ also form an extended complete Chebyšev system and $v_{k} \in C^{l+2}(a, b), k=0, \ldots, l+2$.

A function $f(x)$ on $[a, b]$ is convex with respect to $v_{0}, \ldots, v_{l+1}(l \geq 0)$ if

$$
U\left(\begin{array}{c}
v_{0}, \ldots, v_{l+1}, f \\
x_{0}, \ldots, x_{l+1}, x_{l+2}
\end{array}\right) \equiv\left|\begin{array}{lr}
v_{0}\left(x_{0}\right) & v_{0}\left(x_{l+2}\right) \\
\vdots & \\
v_{l+1}\left(x_{0}\right) \ldots & v_{l+1}\left(x_{l+2}\right) \\
f\left(x_{0}\right) & f\left(x_{l+2}\right)
\end{array}\right| \geq 0
$$

for any $a \leq x_{0}<x_{1}<\ldots<x_{l+2} \leq b$, and we shall write $f \in C\left(v_{0}, \ldots, v_{l+1}\right)$.

For any function $f(x)$ on $(a, b)$ and any set of points $a \leq x_{0}<\ldots<$ $x_{l+1} \leq b$, there exists a unique interpolatory linear function $\phi=\sum_{j=0}^{l+1} a_{j} v_{j}$ s.t. $f\left(x_{j}\right)=\phi\left(x_{j}\right)$ for $j=0,1, \ldots, l+1$.

The following lemmas will be used in the sequel.

Lemma 1. If $\phi$ is linear and interpolates a continuous function $f \in$ $C\left(v_{0}, \ldots, v_{l+1}\right)$ at $a \leq x_{1}<\ldots<x_{l+2} \leq b$, then $\bar{D}^{l} f \leq \bar{D}^{l} \phi$ in some interval $\left(z_{1}, z_{2}\right)$ with $x_{1}<z_{1}<z_{2}<x_{l+2}$.

The proof follows using Theorem 3.1 of [6] and a generalized form of Rolle's theorem.

Lemma 2. If $f \in C\left(v_{0}, \ldots, v_{l+1}\right)$, and $\phi$ is linear and satisfies $\bar{D}^{j} \phi\left(x_{0}\right)=\bar{D}^{j} f\left(x_{0}\right), j=0, \ldots, l+1, a<x_{0}<b$, then $\bar{D}^{j} f(x) \geq \bar{D}^{j} \phi(x)$ for $a \leq$ $x \leq b$.

Use of Lemma 2.2 in [6] and Theorem 3.1 in [5] lead to the proof.

Using these lemmas we can start the study of operators which preserve convexity.

It is possible to reformulate some results of Z. Ziegler [7] and S. Karlin and W. J. Studden [5] in order to obtain a characterization of such operators (see also R. Bojanic and J. A. Roulier [2]).

Theorem 1. Let $T: C[a, b] \rightarrow C[a, b]$ be a continuous linear operator. 
In order that $T: C\left(v_{0}, \ldots, v_{l+1}\right) \rightarrow C\left(v_{0}, \ldots, v_{l+1}\right)(l \geq 0)$ (i.e. a convexity preserving positive (c.p.c.l.) operator), it is necessary and sufficient that

(i) for every linear function $\phi, T(\phi, x)$ is also a linear function,

(ii) $T\left(\phi_{l+1}(t, c), x\right) \in C\left(v_{0}, \ldots, v_{l+2}\right)$ for every $c \in(a, b)$, where

(6) $\phi_{l+1}(t, c)=\left\{\begin{array}{l}w_{0}(t) \int_{x}^{t} w_{1}\left(\zeta_{1}\right) \cdots \int_{x}^{\zeta} w_{i+1}\left(\zeta_{l+1}\right) d \zeta_{l+1} \cdots d \zeta_{1}, x \leq t \leq b, \\ 0, \quad a \leq t<x,\end{array}\right.$

Using a procedure similar to that of P. P. Korovkin [4], it is possible to find a criterion to characterize those sequences of c.p.c.l. operators which converge strongly to the identity and also to find asymptotic relations of Voronskaja's type.

Theorem 2. Let $T_{n}(n \geq 1)$ be a sequence of c.p.c.l. operators and suppose that $\left\|T_{n}\right\| \leq M<\infty$. $T_{n}$ converges strongly to the identity iff

$$
\lim _{n \rightarrow \infty} T_{n} v_{j}=v_{j}, \quad j=0,1, \ldots, l+2 .
$$

Theorem 3. If $f \in C^{(l+2)}(a, b)$ and $T_{n}(n \geq 1)$ is a sequence of c.p.c.l. operators which converges strongly to the identity, then

$$
\lim _{n \rightarrow \infty} \frac{T_{n}(f, x)-f(x)}{T_{n}\left(v_{l+2}, x\right)}=\bar{D}^{l+2} f(x)
$$

and

$$
\lim _{n \rightarrow \infty} \frac{\bar{D}^{l} T_{n}(f, x)-\bar{D}^{l} f(x)}{\bar{D}^{l} T_{n}\left(v_{l+2}, x\right)}=\bar{D}^{l+2} f(x) .
$$

Then when we study the saturation problem it is natural to assume that $T_{n}$ satisfy an asymptotic formula of the following type:

$$
\lim _{n \rightarrow \infty} \lambda_{n} \bar{D}^{l} r_{n}(f, x)=\bar{D}^{l} \rho(x) D f(x), \quad a<x<b,
$$

where $\lambda_{n}>0$ converges to $+\infty, r_{n}(f, x) \equiv T_{n}(f, x)-f(x), \bar{D}^{l} \rho(x) \geq 0$ on $[a, b]$ and $\bar{D}^{l} \rho(x)>0$ on $(a, b)$, and $D$ is a differential operator of type $\bar{D}^{k}$ with $k \geq l+2$. The following result is an extension of [6, Lemma 4.1].

Theorem 4. If $T_{n}(n \geq 0)$ is a sequence of c.p.c.l. operators which satisfies

$$
\lambda_{n} \vec{D}^{l} r_{n}(f, x) \geq-M \bar{D}^{l} \rho(x)+o(1)
$$

with $M \geq 0$, then

$$
f+M v_{l+2} \in C\left(v_{0}, \ldots, v_{l+1}\right)
$$

In the proof of this theorem, Lemma 1 is needed. 
Corollary 1. A sequence of c.p.c.l. operators can satisfy a relation of type (10) only if $k=l+2$. This follows from Theorem 4, choosing $f=$ $-v_{l+2}$ and $M=0$.

Now we state the inverse result to Theorem 4 .

Theorem 5. If $T_{n}(n \geq 1)$ is a sequence of c.p.c.l. operators and $f+$ $M v_{l+2} \in C\left(v_{0}, \ldots, v_{l+1}\right)$, then

$$
\lambda_{n} \bar{D}^{l} r_{n}(f, x)>-M \bar{D}^{l} \rho(x)+o(1) .
$$

This theorem is a simple consequence of Lemma 2 .

Combining Theorems 4,5 and results from $\left[5, \S_{3}\right]$ we find

Corollary 2. A function $f \in C^{l}[a, b]$ satisfies

$$
\lambda_{n}\left|\bar{D}^{l} r_{n}(f, x)\right| \leq M \bar{D}^{l} \rho(x)+o(1), \quad a<x<b,
$$

iff $f \in \operatorname{Lip}_{M} 1$ with respect to $v_{0}, v_{1}, \ldots, v_{l+2}$ (i.e. $\bar{D}^{l+1} f(x)$ is absolutely continuous and $\left.\left|\bar{D}^{l+2} f(x)\right| \leq M[6]\right)$.

Corollary 3. If $M=0$, then if $f$ satisfies a relation of the type $\lambda_{n} \bar{D}^{l} r_{n}(f, x)=o(1)$ for $a<x<b$, then $f$ is linear. This generalizes a result of Bajšanski and Bojanic [1] and al so a remark of [6].

Corollary 4. If $T_{n} v_{j}=v_{j}, j=0,1, \ldots, l+1$, we can remove the $o(1)$ from Corollary 2.

\section{REFERENCES}

1. B. M. Bajšanski and R. Bojanić, A note on approximation for Bernstein polynomials, Bull. Amer. Math. Soc. 70 (1964), 675-677. MR 29 \#3800.

2. R. Bojanic and J. A. Roulier, Approximation of convex functions by convex splines... (to appear).

3. R. DeVore, The approximation of continuous functions by positive linear operators, Springer-Verlag, Berlin and New York, 1972.

4. P. P. Korovkin, Linear operators and the theory of approximation, Fizmatgiz, Moscow, 1959; English transl., Russian Monographs and Texts on Advanced Math. and Phys., vol. 3, Gordon and Breach, New York; Hindustan, Delhi, 1960. MR 27 \#561.

5. S. J. Karlin and W. J. Studden, Tchebycheff systems: With applications in analysis and statistics, Pure and Appl. Math., vol. 15, Interscience, New York, 1966. MR $34 \# 4757$.

6. G. G. Lorentz and L. L. Schumaker, Saturation of positive operators, J. Approximation Theory 5 (1972), 413-424.

7. Z. Ziegler, Generalized convexity cones, Pacific J. Math. 17 (1966), 561 580. MR $34 \# 2804$.

DEPARTMENT OF MATHEMATICS, UNIVERSITY OF ALBERTA, EDMONTON, ALBERTA, CANADA T6G 2G1 